\title{
ORIGINAL
}

\section{RIESGO QUÍMICO LABORAL: ELEMENTOS PARA UN DIAGNÓSTICO EN ESPAÑA}

\author{
Alfonso A Calera Rubio, José María Roel Valdés, Amparo Casal Lareo, Rafael Gadea Merino y \\ Fernando Rodrigo Cencillo
}

Instituto Sindical de Trabajo, Ambiente y Salud.

\section{RESUMEN}

Fundamento: La falta de información junto a la ausencia de un conocimiento preciso de las propiedades intrínsecas de cada agente químico y de la exposición derivada de un uso concreto dificultan en gran medida la prevención de los trabajadores expuestos a los riesgos generados por la presencia de estos productos en los puestos de trabajo. El objetivo del presente estudio es identificar los elementos que permitan efectuar un diagnóstico de la situación del riesgo químico en España y la realización de un inventario de sustancias peligrosas utilizadas en el lugar de trabajo por sectores y/o actividades de producción.

Métodos: Análisis de las fuentes secundarias, identificación de la percepción del riesgo entre representantes de los trabajadores y análisis del tratamiento que recibe el riesgo químico en las evaluaciones de riesgo. Por último se establecen las bases de una Matriz de Exposición a sustancias químicas por sectores de producción.

Resultados: Grandes volúmenes de producción y consumo de agentes químicos, generalización de la multiexposición, pero sin datos sobre su uso concreto, con poca información sobre la peligrosidad intrínseca de las sustancias utilizadas y escasa identificación en las evaluaciones de riesgo. Amplia exposición en los sectores de producción con subregistro del daño a la salud y dificultades en la percepción del riesgo. Elaboración provisional de una matriz de exposición por sectores de producción.

Conclusiones: Se obtienen datos y se identifican fuentes de información y procedimientos de análisis que permiten elaborar un diagnóstico de situación ante riesgo químico laboral. Se obtiene una matriz inicial de exposición por sectores de producción (susceptible de revisión y ampliación en función de la evidencia).

Palabras clave: Riesgo químico laboral, salud laboral, Sustancias Peligrosas, Sustancias, productos y materiales tóxicos. Prevención. Riesgos químicos. Peligros tóxicos. Contaminación ambiental.
ABSTRACT

\section{Occupational Chemical Risk: Elements for a Diagnostic in Spain}

Background: Lack of information and accurate knowledge about the properties of chemicals and the exposure derived from its use; hinder health and safety measures to protect exposed workers. The aim of this study is to identify the elements that will help us draw a balance of chemical hazards in Spain and register the most dangerous chemicals at the workplace (by sector or field of activity).

Methods: Study of secondary sources, identification of risk perception by workers' representatives and study of the approach on chemical risk in risk assessments. The last step is to set up a Chemical Exposure Database arranged by industrial sectors.

Results: Great volumes of production and consumption of chemicals, generalization of exposure, but insufficient data on specific use; little information is provided on specific dangerous factors of commonly used chemicals; chemicals are barely identified in risk assessments. High levels of exposure in industrial sectors; damage is not adequately registered; there are difficulties in risk perception. Set-up of a preliminary database on exposure by sector of activity.

Conclusions: Data is collected; information sources and procedures, which allow a forecast of chemical risk, are identified. A database on exposure by sector of activity is developed (to be checked and enhanced as new evidence is found).

Key words: Chemical contamination. Toxic Substances, Products and Materials. Toxic dangers. Occupational health. Environmental pollution.

Correspondencia:

Alfonso A. Calera Rubio

ISTAS

C/ Cardenal Belluga 10, Entrlo

03005 Alicante. 


\section{INTRODUCCIÓN}

De los más de 25 millones de sustancias químicas existentes en el mundo ${ }^{1}$, en la Unión Europea (UE) se comercializan y están registradas 100.195, de las que las empresas utilizan habitualmente unos 30.000 , a pesar de que 20.000 no han sido objeto de pruebas toxicológicas completas y sistemáticas y que el $21 \%$ de las sustancias químicas de alto volumen de producción (más de 1000 T/año) no disponen de datos toxicológicos ${ }^{2}$. De las sustancias químicas registradas que presentan efectos toxicológicos conocidos 350 son cancerígenas y 3.000

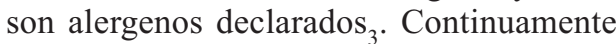
se introducen nuevas sustancias de toxicidad mal conocida por la insuficiente información científica en relación con la posibilidad de efectos nocivos sobre el medio ambiente y la salud a medio y largo plazo.

El riesgo generado por la falta de información ${ }^{4-6}$ se ve acrecentado por el insuficiente cumplimiento de la normativa, las malas prácticas, los errores en el etiquetado y las fichas de datos de seguridad facilitadas por los fabricantes ${ }^{7,8}$, falta de formación o dificultades para interpretar el etiquetado ${ }^{9}$ por parte de los trabajadores y usuarios, el uso combinado de sustancias diferentes -riesgo por multiexposición-, mezclas de agentes químicos realizadas en la propia empresa, precariedad laboral ${ }^{10}$, tóxicos ya existentes en el lugar de trabajo (espacios confinados), generados en otras actividades productivas (humos de motores diesel) o la aparición de nuevas sustancias por reacción de las existentes, entre otras.

Los colectivos que están más expuestos a esta contaminación química son, lógicamente, quienes más próximos están a la fuente, esto es, los trabajadores de prácticamente todos los sectores, en diversos grados. Se estima que el porcentaje de trabajadores europeos expuestos a agentes químicos es del 20-44 \% del total ${ }^{11,12}$.
Las enfermedades degenerativas derivadas de la exposición habitual a agentes químicos, son con mucho, más importantes y frecuentes que los accidentes de trabajo. A nivel mundial la Organización Internacional del Trabajo (OIT) estima que de los 2 millones de muertes laborales que tienen lugar cada año en el mundo, 440.000 se producen como resultado de la exposición de trabajadores a agentes químicos ${ }^{13}$. En la Unión Europea se considera que se producen anualmente 32.000 muertes por cáncer, 16.000 enfermedades cutáneas, 6.700 enfermedades respiratorias, 500 enfermedades oculares y 570 enfermedades del SNC ${ }^{11}$.

La falta de información, la ausencia de un conocimiento preciso de las propiedades intrínsecas de una sustancia, de la exposición derivada de un uso concreto y de su eliminación (requisito previo indispensable para la toma de decisiones relativa a la gestión segura de las sustancias químicas) dificulta en gran medida la prevención de los trabajadores expuestos a los riesgos derivados del uso de agentes químicos en los puestos de trabajo y explica buena parte de los daños a la salud ${ }^{14}$.

El objetivo del presente estudio es identificar los elementos que permitan realizar un diagnóstico de la situación del riesgo químico en España: fuentes de información de uso, exposición y daño, percepción del riesgo y análisis del tratamiento que recibe el riesgo químico en las evaluaciones de riesgo realizadas en las empresas, con el fin de estudiar propuestas de actuación de cara a mejorar las condiciones de seguridad e higiene, como es el inventario de sustancias peligrosas utilizadas en el lugar de trabajo por sectores de producción.

\section{SUJETOS Y MÉTODOS}

Se utiliza como estrategia de investigación la conjunción de técnicas, compaginando los datos estadísticos y documentales dis- 
ponibles junto a la producción de información mediante diferentes técnicas de investigación.

\section{Revisión bibliografía/fuentes secundarias (datos para España)}

Para la obtención de los datos de producción, consumo y uso, se han identificado las siguientes fuentes útiles:

- Encuesta Anual Industrial de Productos (EAIP $)^{15}$ realizada por el Instituto Nacional de Estadística (INE 20002001), en ella se hace una valoración individualizada de los principales productos industriales españoles definidos mediante la lista de productos PRODCOM (PRODucción COMunitaria), recoge la producción anual de unos 400 productos químicos, metales y minerales de un total de 5000 productos.

- Base de Datos de Comercio Exterior perteneciente al Consejo Superior de Cámaras y Aduanas de la Agencia Tributaria $^{16}$, permite obtener información sobre importaciones y exportaciones españolas, de productos de la industria química o las industrias conexas por peso y valor. Analizadas para el 2003.

- Federación Empresarial de la Industria Química Española (FEIQUE), emite informes anuales sobre el sector: valor de la producción, distribución por sectores, distribución geográfica, estructura, comercio exterior y empleo. Se han utilizado datos procedentes de los años 2002 al $2004^{17}$.

La exposición laboral se ha definido a partir de las III ${ }^{18}$, IV ${ }^{19}$ y V $^{20}$ Encuesta Nacional de Condiciones de Trabajo que confecciona el Instituto Nacional de Seguridad e Higiene en el trabajo; el Informe Nacional de España del Estado de la Seguridad y la Salud en la Unión Europea de Junio 1999²1, base de datos CAREX (Carcinogen Exposure) ${ }^{22-24}$ y el informe de la Secretaría de Salud Laboral de la USMR-CC.OO sobre Exposición laboral a agentes cancerígenos y mutágenos ${ }^{25}$ a través de visitas efectuadas en 222 empresas.

Los indicadores de daño a la salud se han obtenido analizando las estadísticas oficiales de daño laboral:

- Accidentes de trabajo por agentes químicos durante los años $1999^{26}, 2000$, $2001^{27}$ y $2002^{28}$. Los procesos tóxicos por agentes químicos se declaran fundamentalmente como accidentes de trabajo (AT) dentro de diferentes epígrafes que se recogen en este sistema de registro, bajo el código: de forma Exposición a sustancias nocivas, contactos con sustancias cáusticas o corrosivas y explosiones para el año 1999 o bajo el código agente material causante Químicos, Explosivos (2000-2001), a los que podríamos añadir buena parte de los casos presentes en el código polvo -básicamente sílices y sensibilizantes-.

- Las enfermedades degenerativas derivadas de la exposición habitual a agentes químicos para los años 2000 , $2001^{29}, 2002,2003^{30}$, aparecen reflejadas como «Enfermedades Profesionales producidas por agentes químicos».

La percepción del riesgo se analiza a partir de la V Encuesta Nacional de condiciones de trabajo ${ }^{20}$ y mediante un cuestionario específico que recogía entre otras cuestiones información sobre los riesgos laborales percibidos en las empresas. Se obtienen 1.540 encuestas pertenecientes a responsables sindicales y delegados de prevención del sindicato $\mathrm{CCOO}$ procedentes de todo el país, en junio del 2003 en Madrid aprovechando una asamblea nacional. Todas las preguntas del cuestionario eran cerradas, debiendo señalar los encuestados sobre un listado de posibles respuestas para cada aspecto evaluado un máximo de tres. En casi todas las preguntas 
se dejaba también opción a una respuesta abierta bajo la categoría de «Otros». La codificación y registro de la información contenida en los cuestionarios fueron realizados por una empresa especializada. El análisis de los datos fue llevado a cabo con el programa Stata (v.7.0).

\section{Elaboración de una matriz de exposición a sustancias químicas por sectores de producción, en cuatro etapas}

En la primera etapa se desarrolla mediante dos procedimientos: a) a partir de la bibliografía consultada ${ }^{31-34}$ se obtiene un primer listado de productos utilizados según actividades económicas (se utilizan los códigos de las actividades económicas correspondientes al CNAE-1993 REV1, con dos o más dígitos, en función de la actividad) con riesgo de exposición a sustancias químicas y para las que se dispone de datos; b) consulta a 23 expertos sobre la matriz inicial. En una segunda etapa se completa con las sustancias utilizadas en diferentes actividades económicas a partir de una encuesta realizada en la región de Alsacia (Francia) por la Caisse Regionale d'Assurance Maladie AlsaceMoselle $^{35}$. Tercera etapa: se añaden al listado los potenciales efectos en la salud humana de estas sustancias, a partir de la Base de datos UCLID CD-ROM. Year 2000 Edition. Public data on high volume chemicals. Y del EUESIS (EUropean Existing Substances Information System). La calificación de las propiedades carcinogénicas se ha obtenido a partir de clasificación de las sustancias cancerígenas que hace el Real Decreto 363/1995 sobre notificación de sustancias nuevas y clasificación, envasado y etiquetado de sustancias peligrosas, en su Anexo1, de los RD 665/1997 y 1124/2000 sobre agentes cancerígenos, de los listados de sustancias cancerígenas de la International Agency for Research on Cancer (IARC) $)^{36}$ y de la American Conference of Governmental Industrial Hygienists (ACGIH) ${ }^{37}$. La cali- ficación de los efectos en diversos órganos, aparatos y/o sistemas: cardiocirculatorio, riñón, respiratorio, piel y órganos de los sentidos, nervioso, inmunitario, hígado y gastrointestinal, endocrino, y reproductivo, se ha obtenido a partir de la base de datos Health effects de SCORECARD ${ }^{38}$. Cuarta etapa: Validación-Ajuste de la matriz. Se ha validado parcialmente por diferentes procedimientos: mediante trabajo de campo por equipo de técnicos de Seguridad e Higiene de la Organización Territorial de ASEPEYO y de ISTAS-CCOO, y mediante consulta a expertos e instituciones para diferentes sectores.

\section{Análisis de las evaluaciones de riesgos de empresas}

Apoyados en un informe previo realizado por la Secretaría de Salud Laboral de USMR-CCOO ${ }^{39,40}$, se analizaron 59 evaluaciones procedentes de empresas situadas en las siguientes provincias y/o CC.AA.: Alicante, Castilla la Mancha, Madrid, Mallorca y Valencia. Se comparan los datos que aportan las evaluaciones de riesgos sobre los riesgos por agentes químicos con los criterios y las necesidades de información que establece el Real Decreto 374/2001 y la Guía Técnica para la evaluación y prevención de los riesgos presentes en los lugares de trabajo relacionados con agentes químicos. Previamente se elaboraron los correspondientes «ítems» que componen el cuestionario, incluyéndose aspectos relativos a las características concretas de la empresa y a la entidad que ha realizado la evaluación de riesgos.

\section{RESULTADOS}

\section{Producción, consumo y uso de agentes químicos}

La industria química nacional en los últimos cinco años ha registrado un crecimiento 
Tabla 1

Exportaciones durante todo el año de 2003 referentes a la sección: «Productos de las industrias quimicas o de las industrias conexas». Por capítulo, ordenados por peso. Base de Datos de Comercio Exterior. Consejo Superior de Cámaras y Aduanas (Agencia Tributaria)

\begin{tabular}{|l|r|r|r|}
\hline \multicolumn{1}{|c|}{ CAPÍTULO } & \multicolumn{1}{|c|}{$\begin{array}{c}\text { Peso } \\
\text { (Miles de } \\
\text { Kgs. }\end{array}$} & \multicolumn{1}{|c|}{$\begin{array}{c}\text { Valor } \\
\text { (Miles de } \\
\text { euros) }\end{array}$} & \multicolumn{1}{|c|}{$\begin{array}{c}\text { Número de } \\
\text { operaciones }\end{array}$} \\
\hline $\begin{array}{l}\text { [28] PTOS.QUIMICOS INORGANICOS; COMPUESTOS INORGANICOS } \\
\text { U ORGANICOS DE METALES PRECIOSOS,ELEMENTOS } \\
\text { RADIACTIVOS }\end{array}$ & 2.781 .021 & 469.214 & 24.952 \\
\hline [29] PRODUCTOS QUIMICOS ORGANICOS. & 1.912 .656 & 2.874 .976 & 83.720 \\
\hline [31] ABONOS. & 1.326 .311 & 201.962 & 8.863 \\
\hline $\begin{array}{l}\text { [32] EXTRACTOS CURTIENTES O TINTOREOS; TANINOS Y } \\
\text { DERIVADOS; PIGMENTOS COLORANTES;PINTURAS Y } \\
\text { BARNICES;MASTIQUES;TINTAS }\end{array}$ & 1.102 .070 & 1.338 .126 & 105.774 \\
\hline [38] PRODUCTOS DIVERSOS DE LAS INDUSTRIAS QUIMICAS & 906.507 & 1.250 .001 & 62.031 \\
\hline $\begin{array}{l}\text { [34] JABONES,CERAS,PTOS.DE LIMPIEZA,PASTAS PARA } \\
\text { MODELAR,PREPARACIONES ODONTOLOGICAS }\end{array}$ & 840.292 & 917.655 & 71.811 \\
\hline $\begin{array}{l}\text { [33] ACEITES ESENCIALES Y RESINOIDES; PREPARACIONES DE } \\
\text { PERFUMERIA,TOCADOR O COSMETICA }\end{array}$ & 241.987 & 1.308 .704 & 89.141 \\
\hline [30] PRODUCTOS FARMACEUTICOS & 89.130 & 3.432 .149 & 46.211 \\
\hline $\begin{array}{l}\text { [35] MATERIAS ALBUMINOIDEAS; PTOS.A BASE DE ALMIDON O } \\
\text { FECULAS MODIFICADAS;COLAS;ENCIMAS }\end{array}$ & 55.121 & 102.027 & 18.205 \\
\hline [37] PRODUCTOS FOTOGRAFICOS O CINEMATOGRAFICOS. & 40.774 & 181.435 & 12.361 \\
\hline $\begin{array}{l}\text { [36] POLVORAS Y EXPLOSIVOS; ARTICULOS DE PIROTECNIA; } \\
\text { CERILLAS; ALEACIONES PIROFORICAS;MAT.INFLAMABLES }\end{array}$ & 3.945 & 23.891 & 1.306 \\
\hline T O T A L & 9.299 .813 & 12.100 .140 & 524.375 \\
\hline
\end{tabular}

acumulado del $36 \%$ y un crecimiento del consumo de un $34 \%$. España importa unos 12 millones de toneladas y exporta algo más de 9 , con lo que se puede estimar que el consumo anual se sitúa en unos 24 millones de toneladas de productos químicos (tabla 1).

El 86,4 \% de las más de 3.789 empresas que operan en el sector tienen menos de 50 trabajadores en plantilla, siendo nuestro país, junto a Italia, el que mayor número de pequeñas y medianas empresas (PYMES) integra, el 56,2\% con 10 o menos trabajadores, en su mayoría con escaso o ningún conocimiento experto en toxicología.

Se constata el uso combinado de muchas sustancias, la existencia de riesgo por multiexposición, y el desconocimiento de sus efectos. Múltiples y variadas exposiciones a productos tóxicos como consecuencia de la precariedad laboral y el cambio frecuente de empleo.

\section{Niveles de exposición por actividades laborales y por sectores}

No existen datos cuantitativos sobre prevalencia y niveles de exposición a agentes químicos por puesto de trabajo o por sectores de producción, ya que algunas fuentes naturales de información, como las evaluaciones de riesgo, no suelen contener estos datos. Tampoco existen estudios ad hoc actualizados sobre exposición de los trabajadores a determinados productos o a niveles sectorial o de actividad económica.

Todos los sectores están expuestos a sustancias peligrosas en diversos grados, pero los que presentan mayores riesgos, según la Agencia Europea para la Seguridad y la salud, son agricultura, industria química, limpieza, construcción, tratamiento de alimentos, peluquería, asistencia sanitaria, mecánica/talleres de automóviles, impre- 
sión, textil/curtidos, recogida y tratamiento de residuos ${ }^{3}$.

E1 31\% de los trabajadores está expuesto a contaminantes químicos, ya sea por inhalación de polvos, humos, vapores, gases, o por manipulación de productos. El 19\% manipula en su puesto productos nocivos o tóxicos. La manipulación es más frecuente en el sector industria $(27,8 \%)$ y dentro de este sector en la rama química. El 22,3\% de los trabajadores respira en su puesto de trabajo polvos, humos, gases o vapores nocivos o tóxicos. La inhalación es más frecuente en el sector construcción (50,6\%) aunque está muy cercano el sector industria $(37,4 \%)$. Los trabajadores no cualificados $(25,5 \%)$ y los oficiales, operarios y artesanos $(23,2 \%)$ son los trabajadores con mayor porcentaje de expuestos. Además, la exposición a este riesgo muestra una tendencia ascendente en los últimos 3 a 5 años. Por tipo de contrato son los trabajadores cedidos por empresas de trabajo temporal los más expuestos $(55,6 \%)$.

Las fuentes de datos disponibles apuntan a que la exposición de los trabajadores a las sustancias químicas más peligrosas (CMR, disruptores, bioacumulativas) está muy extendida. La exposición a sustancias peligrosas como los cancerígenos es especialmente preocupante, tanto por la gravedad del riesgo como por la población expuesta. Se ha estimado (CAREX) que más de tres millones de trabajadores españoles han estado expuestos a estas sustancias. El estudio de la Secretaría de salud laboral de CCOO en la Comunidad de Madrid indica un importante uso de agentes cancerígenos en las empresas, más de 2 de media. La matriz de exposición, elaborada para este estudio (tabla 2), identifica un importante número de estas sustancias en diferentes actividades económicas: 53 en la industria química, 31 en la fabricación de otros productos minerales no metálicos, 27 en la fabricación de productos metálicos y 17 en la industria de alimentos y bebidas. Este método ha permitido identificar, asimismo, el uso de gran número de otras sustancias peligrosas (embriotóxicas, tóxicas para la reproducción, disruptores endocrinos, neurotóxicas, y sensibilizantes) en las diferentes actividades económicas.

Sobre la gestión de la prevención: a) La participación de los trabajadores en la gestión del riesgo químico en las empresas es

Tabla 2

Análisis de la presencia de las sustancias especialmente peligrosas en algunas de las principales actividades económicas

\begin{tabular}{|c|c|c|c|c|c|c|c|}
\hline SECTOR/ACTIVIDAD & $\mathbf{N}$ & 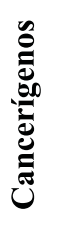 & 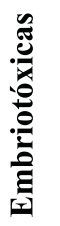 & 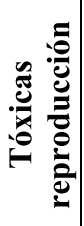 & 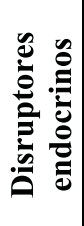 & 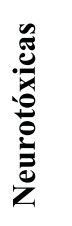 & 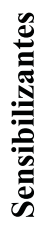 \\
\hline Industria química & 603 & 53 & 31 & 23 & 40 & 165 & 56 \\
\hline $\begin{array}{l}\text { Fabricación de otros productos } \\
\text { minerales no metálicos }\end{array}$ & 274 & 31 & 17 & 7 & 27 & 71 & 44 \\
\hline Fabricación de productos metálicos & 270 & 27 & 11 & 5 & 19 & 106 & 33 \\
\hline Industria de alimentos y bebidas & 236 & 17 & 10 & 5 & 18 & 90 & 15 \\
\hline
\end{tabular}


escasa y cuando esta tiene lugar es una participación de carácter puntual. b) En las evaluaciones de riesgo la información relativa al riesgo químico es de muy baja calidad. No se evalúan todos los puestos de trabajo o no identificar a todos los trabajadores expuestos o las tareas que estos realizan o las sustancias químicas presentes. No se tienen en cuenta los datos de salud de los trabajadores ni las características especiales de los trabajadores (sensibles, jóvenes, mujeres, con problemas de salud...), solamente en algunas empresas grandes se tiene en cuenta esta información y se evidencia que en su tratamiento existe un claro sesgo a favor de los aspectos de la seguridad. c) La actuación preventiva frente al riesgo químico en las empresas es, por lo general, de carácter puntual y reactivo ante accidentes e incidentes. Predominan las medidas de protección individual frente a las colectivas y cuando éstas existen suelen presentar problemas de adecuación y mantenimiento. La eliminación y la sustitución de las sustancias origen del riesgo no constituye el objetivo prioritario en las empresas. d) La vigilancia de la salud es un aspecto de la actividad preventiva que no suele integrarse ni en la evaluación de riesgos ni en el conjunto de la gestión preventiva de la empresa.

Tabla 3

Accidentes de trabajo con baja, por agente material causante

\begin{tabular}{|l|r|r|r|}
\hline \multicolumn{1}{|c|}{ AÑO } & $\mathbf{2 0 0 0}$ & $\mathbf{2 0 0 1}$ & $\mathbf{2 0 0 2}$ \\
\hline ACCIDENTES DE TRABAJO POR: & & & \\
• Agentes químicos & 9.697 & 9.665 & 8.868 \\
• Agentes explosivos & 636 & 719 & 694 \\
• Agentes físicos. Polvo & 9.900 & 8.938 & 8.705 \\
\hline TOTAL & 20.233 & 19.322 & 18.267 \\
\hline
\end{tabular}

Tabla 4

Enfermedades profesionales por tipo de enfermedad

\begin{tabular}{|c|c|c|c|c|}
\hline AÑO & 2000 & 2001 & 2002 & 2003 \\
\hline ENFERMEDADES PROFESIONALES: & & & & \\
\hline - Por agentes químicos & 361 & 480 & 371 & 433 \\
\hline $\begin{array}{l}\text { - De la piel por agentes no incluidos } \\
\text { anteriormente }\end{array}$ & 2.043 & 2.084 & 1.969 & 2.079 \\
\hline $\begin{array}{l}\text { - Por inhalación de agentes no incluidos } \\
\text { anteriormente }\end{array}$ & 450 & 521 & 515 & 567 \\
\hline $\begin{array}{l}\text { - Enfermedades sistémicas por agentes } \\
\text { químicos }\end{array}$ & 6 & 7 & 14 & 12 \\
\hline TOTAL & 2.860 & 3.092 & 2.869 & 3.091 \\
\hline
\end{tabular}




\section{Evaluación del daño a la salud}

En las estadísticas oficiales de daño laboral, los procesos tóxicos por agentes químicos se declaran fundamentalmente como accidentes de trabajo (AT) dentro de diferentes epígrafes que se recogen en este sistema de registro. Las enfermedades degenerativas derivadas de la exposición habitual a agentes químicos aparecen reflejadas como «Enfermedades Profesionales producidas por agentes químicos» en el apartado A de la lista vigente de enfermedades profesionales. En las tablas 3 y 4 se muestran el número de accidentes por agentes químicos entre el año 2000 y el 2002 y las enfermedades profesionales del 2000 al 2003. Estos accidentes supusieron alrededor del $2 \%$ del total de los accidentes registrados con baja para el año 2001 y también para el 2002.

No obstante, al menos en el caso de las enfermedades, estas cifras no reflejan la realidad. El subregistro de enfermedades ocasionadas por agentes químicos en el trabajo se puede valorar acudiendo a la comparación entre el volumen de enfermedades registradas como enfermedades profesionales y el volumen de enfermedades estimadas en base a estudios epidemiológicos ${ }^{41,42}$. En la tabla 5 se presenta la estimación de la mortalidad para las principales enfermedades por exposición a sustancias tóxicas.

\section{Sobre la percepción y el conocimiento del riesgo}

Los sujetos más interesados en la prevención, los empresarios y los propios trabajadores, tienen un escaso conocimiento del riesgo de exposición a los agentes químicos. Este desconocimiento se extiende a los riesgos derivados de la exposición a tóxicos muy peligrosos como los agentes cancerígenos o mutágenos ${ }^{24}$.

Cuando en la V Encuesta Nacional de Condiciones de Trabajo se les pregunta por lo que consideran como unas condiciones molestas o muy molestas, la percepción de los contaminantes ocupa el decimotercer lugar, de un total de una lista de catorce riesgos. La percepción del riesgo para la salud está relacionada con el efecto inmediato, con el accidente, entendiendo éste como el hecho sorpresivo, violento y de consecuencias visibles y traumáticas en el momento de producirse.

En la encuesta realizada por ISTAS a delegados de prevención de CCOO en la que se les preguntaba sobre los riesgos que consideraban principales en su empresa, el riesgo por exposición a productos tóxicos se encuentra en séptimo lugar entre los riesgos que mayor preocupación suscitan en los sectores industriales adquiriendo mayor importancia si se añade el riesgo percibido en ambientes con polvo. En los servicios es percibido de forma secundaria frente a otros riesgos. Existen pequeñas diferencias entre hombres y mujeres al identificar estas últimas el riesgo tóxico entre los cinco primeros.

\section{Elaboración de una Matriz de Exposición a sustancias químicas por sectores de producción}

Un ejemplo de la información resultante puede apreciarse en las figuras 1 y 2 .

\section{DISCUSIÓN}

El abordaje múltiple utilizado ha permitido obtener datos e identificar gran variedad de fuentes de información -sus limitaciones, sus deficiencias- y procedimientos de análisis que facilitan la elaboración de un diagnóstico de situación ante riesgo químico laboral.

La producción y consumo de sustancias peligrosas son una fuente de información indirecta, a mayor producción y consumo 
Tabla 5

Estimación de muertes por las principales enfermedades y lesiones por exposición laboral a sustancias tóxicas en España, 1999

\begin{tabular}{|lcc|}
\hline Condición & $\begin{array}{c}\text { Tasas por millón } \\
\text { asalariados }\end{array}$ & $\begin{array}{c}\text { Muertes por } \\
\text { exposición laboral }\end{array}$ \\
\hline Cáncer & 97 & 1.116 \\
Pulmón & 57 & 656 \\
Mesotelioma & 14 & 161 \\
Leucemia & 6 & 69 \\
Vejiga & 4 & 46 \\
Enfermedades mayores & 68 & 782 \\
Cardiovasculares & 61 & 702 \\
Asma & 3 & 35 \\
Enfermedades por inhalación de polvo & 4 & 46 \\
Asbestosis & 2 & 23 \\
Silicosis & 1 & 12 \\
Intoxicaciones agudas & 3 & 35 \\
Total & & 1.979 \\
\hline
\end{tabular}

mayor será la exposición de los trabajadores, del medio ambiente y de la población general. La única fuente de información oficial sobre este tema es la Encuesta Anual Industrial de Productos (EAIP) que recoge la producción de unos 400 productos químicos, metales y minerales. Sin embargo los productos más peligrosos están bajo secreto estadístico, de hecho, sólo son públicos los datos de 3 de las 18 sustancias cancerígenas que aparecen (INE, 2003).

La preocupante falta de datos sobre producción, consumo y usos de sustancias químicas en los diferentes sectores y actividades económicas hacía muy difícil estimar la magnitud del problema, lo que generó la necesidad de elaborar una matriz inicial de exposición por sectores de producción (susceptible de revisión y ampliación en función de la evidencia), que esperamos ir completando con la colaboración de todos los interesados.
Por motivo de espacio en este trabajo sólo se ha presentado una parte de los procedimientos utilizados para identificar la percepción del riesgo en los colectivos implicados (técnicos de prevención, empresarios, delegados de prevención, trabajadores). No se presentan los resultados de los grupos de discusión con trabajadores, las entrevistas en profundidad con expertos, el análisis de la legislación específica o de los convenios colectivos.

Las estadísticas oficiales muestran solamente la punta del iceberg del daño producido por el uso sustancias químicas en la producción y el comercio. El uso y manipulación de sustancias químicas supone un riesgo no despreciable para la seguridad de los trabajadores, pero sobre todo produce un importante daño a la salud no reconocido. Los usuarios, empresarios y trabajadores tienen un gran desconocimiento de los daños ocasionados por la exposición a sustancias 
Figura 1

Matriz de Exposición a sustancias químicas por sectores de producción. Disponible en: http://www.istas.net

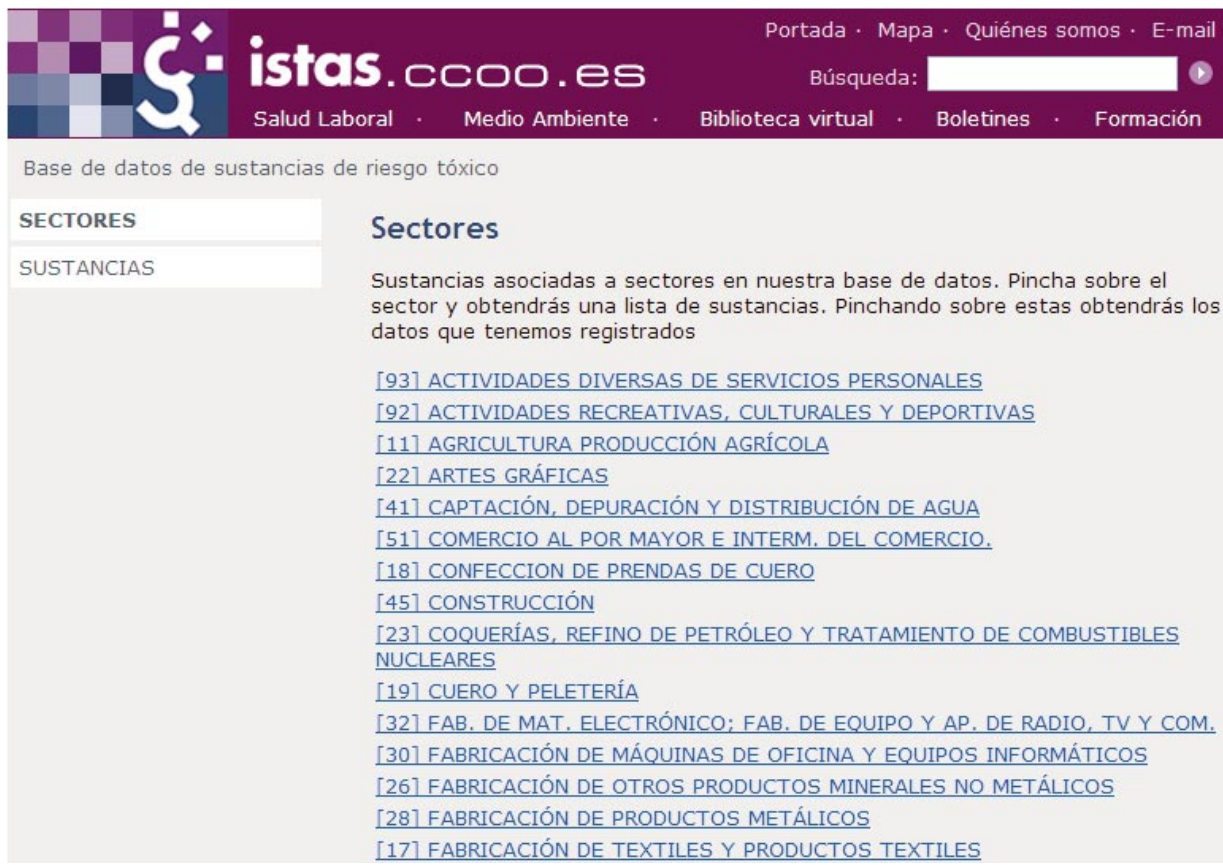

químicas y muestran una escasa sensibilidad frente a este problema.

Las fuentes de datos disponibles apuntan a que la exposición de los trabajadores a las sustancias químicas más peligrosas -cancerígenos, mutágenos, tóxicos para la reproducción (CMR)-, está muy extendida y no se identifica en las evaluaciones de riesgo.

En las evaluaciones de riesgo la información relativa al riesgo químico es de muy baja calidad y se evidencia que en su tratamiento existe un claro sesgo a favor de los aspectos de la seguridad. La vigilancia de la salud es un aspecto de la actividad preventiva que no se integra ni en la evaluación de riesgos ni en el conjunto de la gestión preventiva de la empresa.

El cuadro normativo referido al control del riesgo químico es demasiado complejo y necesita ser clarificado. Por otro lado, se hace necesaria una mayor integración de las vertientes de salud laboral, medio ambiente y salud pública en la normativa.

Los retos ante los que nos encontramos son muchos y variados: a) conseguir que las instituciones elaboren y hagan pública información fiable y sistematizada sobre la producción, consumo, uso y emisiones al medio ambiente de sustancias químicas, b) conseguir que empresarios y trabajadores dispongan de información veraz y entendible sobre los riesgos de las sustancias químicas presentes en los lugares de trabajo, c) conseguir que los órganos de la administración elaboren y publiquen información fiable sobre exposición laboral y ambiental a las sustancias químicas más peligrosas y promuevan estudios sobre riesgos de la multiexposición. 
Figura 2

Matriz de Exposición a sustancias químicas por sectores de producción. Página de información sobre una sustancia específica. Disponible en: http://www.istas.net

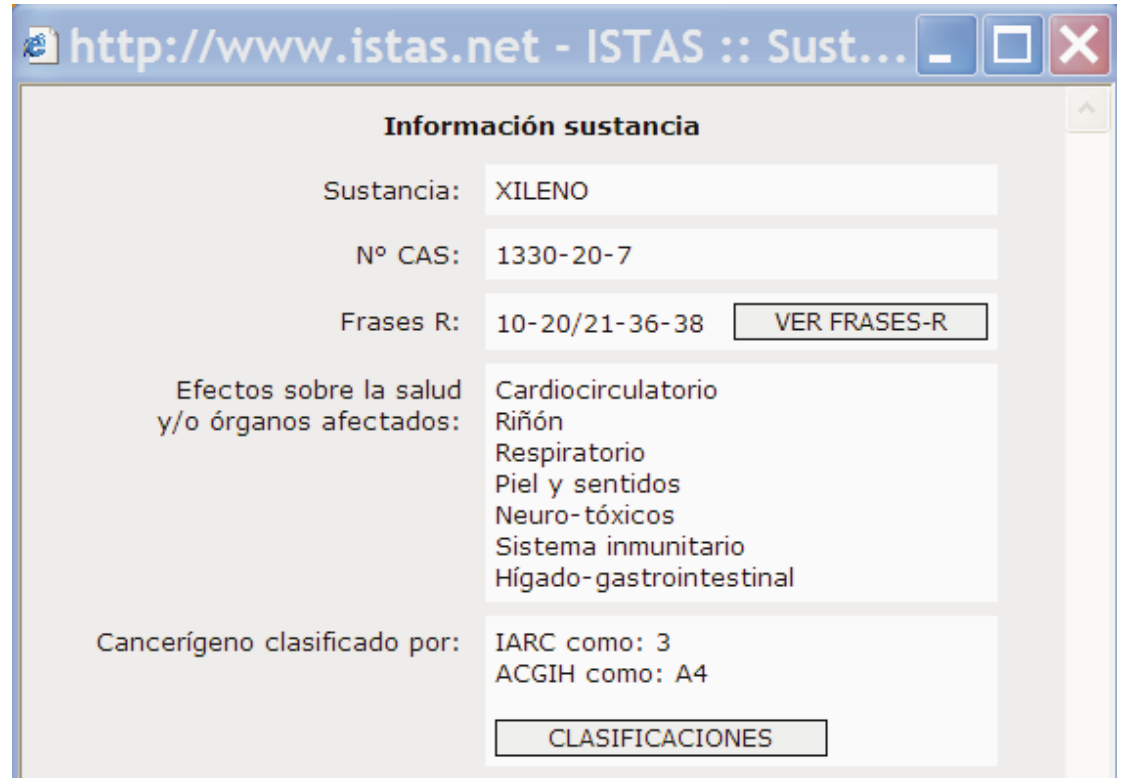

\section{AGRADECIMIENTOS}

A la dirección de ASEPEYO. MATEPSS $\mathrm{N}^{\mathrm{o}} 151$ por apoyar y financiar este proyecto, a Santos Huertas Ríos (Área Higiene Agentes Químicos de la DSH) y Técnicos de $\mathrm{SH}$ de la Organización Territorial.

En el equipo de ISTAS a las compañeras que han participado en el diseño de la metodología de investigación y en la obtención de resultados como: Ana M ${ }^{\text {a }}$ García, Clara LLorens, María José López Jacobs, Ana García, Dolores Romano y Agustín González.

\section{BIBLIOGRAFÍA}

1. American Chemical Society. The Latest CAS Registry Number and Subtance Count. [citado 30 de dic. 2004]. Disponible en: http://www.cas. org/cgi-bin/regreport.pl.
2. Allanou R, et al. Public Availability of Data on EU High Production Volume Chemicals, October 2002. [citado 20 Sept 2003].

3. European Agency for Safety and Health at Work. Sustancias Peligrosas. Mucho Cuidado. Semana Europea para la Seguridad y Salud en el Trabajo; 2003 Mayo. [citado 20 de sep. 2004]. Disponible en: http://osha.eu.int/ew2003/presspack/pack es.doc

4. Intergovernmental Forum on Chemical Safety, Third Session, Forum III Final Report. Annex 6: Priorities for Action Beyond 2000. [citado 20 Sept 2003]. Disponible en: www.who.int/ifcs/Documents/Forum/ForumIII/f3-finrepdoc/Priorities.pdf

5. International Register of Potentially Toxic Chemicals: The quest for Chemical Safety. IRPTC Bulletin (1985) 7:1-2.

6. National Research Council. Toxicity Testing: Strategies to Determine Needs and Priorities. Washington DC: National Academy Press; 1984.

7. Comisión Europea. Three Yearly Report on the Implementation of the Directive 67/548/EEC on 
the Classification, Packaging and Labelling of Dangerous Substances, as Amended by Directive 92/32/EEC, Bruselas: Comisión Europea; 1998 mayo; 62-64. Disponible en: http:/europa.eu.int/ comm/environment/dansub/3year-rep.pdf

8. Fatás Juberias B. Participación de las Comunidades Autónomas en los proyectos europeos de control de productos químicos. En: Sociedad Española de Sanidad Ambiental. $7^{\text {a }}$ Jornada Técnica SESA. Seguridad Química; 2000 Jun 6; Madrid, España; p. 17-19

9. Arbetsmiljöfondens. Hur förstås varningssymboler och risk/skyddsfraser? Arbetsmiljöfondens Sammanfattiningar . Stockholm 1988: 1207

10. Calera Rubio A, Blount E, Riechmann Fernández J. Riesgo Químico: Estrategias de Intervención Social. Rev Salud Ambient 2002;2(1): 48-52

11. Risk \& Policy Analysts Limited. Assessment of the Impact of the New Chemicals Policy on Occupational Health, Final report prepared for European Commission - Environment Directorate-General; 2003 March. [citado 20 de sep. 2004]. Disponible en: http://europa.eu.int/comm/environment/chemicals/pdf/finrep_occ_health.pdf

12. Pascal P, Merllié D. Third European survey on working conditions 2000. Dublin (Ireland): European Foundation for the Improvement of Living and Working Conditions; 2001. Disponible en: http://www.eurofound.eu.int/publications/files/EF 0121EN.pdf

13. Takala Y. OIT. Intervención en la reunión de Enfoque Estratégico para una Gestión Química Internacional (SAICM). PrepCom1, 9-13 Noviembre 2003. Bangkok.

14. Comisión de las Comunidades Europeas. Libro Blanco. Estrategia para la futura política en materia de sustancias y preparados químicos. COM(2001) 88 final. Bruselas; 2001. Disponible en:: http//europa.eu.int/comm/environment/chemicals/whitepaper.htm

15. Instituto Nacional Estadística. Encuesta Industrial de productos.. Disponible en: http://www.ine.es/ pls/eip/eip_entrada

16. Consejo Superior de Cámaras y Aduanas (Agencia Tributaria). Base de Datos de Comercio Exterior. Disponible en: http://aduanas.camaras.org/

17. Federación Empresarial de la Industria Química Española. Radiografía del Sector Químico. Madrid: Feique; 2004. [citado 30 de dic. 2004].
Disponible en: http://www.feique.org/comunica/ rad20047.pdf

181 INSHT. III Encuesta Nacional de Condiciones de Trabajo. 1997, Disponible en: http://www.mtas. es/insht/statistics/enct_3.htm

19. INSHT. IV Encuesta Nacional de Condiciones de Trabajo. 2000, Disponible en: http://www.mtas.es/ insht/statistics/enct_4.html

20. INSHT. V Encuesta Nacional de Condiciones de Trabajo. 2004, Disponible en: http://www.mtas.es/ insht/statistics/enct_5.htm

21. Ministerio de Trabajo y Asuntos Sociales. El Estado de la Seguridad y la Salud en la Unión Europea. Informe Nacional de España. Junio 1999. Pag 3745. Disponible en: http://www.mtas.es/insht/statistics/inf_osh.pdf

22. Kogevinas, M.; Maqueda, J.; de la Orden, V.; Fernández-Gómez, F.; Kauppinen, T.; Benavides, F.G. Exposición a carcinógenos laborales en España: aplicación de la base de datos CAREX. Arch Prev Riesgos Labor. 2000. 3: 153-159.

23. Maqueda, J. et al. CAREX. Occupational exposure to carcinogens in Spain in 1990-93. Helsinki: Finnish Institute of Occupational Health; 1998.

24. 19: Gonzalez CA, Agudo A. Occupational cancer in Spain. Environ Health Perspect 1999;107 Suppl 2:273-7.

25. Mancheño C, Izquierdo MA y col. Secretaría de Salud Laboral de la USMR-CCOO Exposición laboral a agentes cancerígenos y mutágenos. Ediciones GPS-Madrid; 2003

26. Ministerio de Trabajo y Asuntos Sociales. Registro de Accidentes de Trabajo y Enfermedades Profesionales ATE-38-39-40. Epígrafes: enfermedades profesionales producidas por agentes químicos, de la piel producidas por agentes no incluidos anteriormente, por inhalación de sustancias y agentes no incluidos anteriormente y enfermedades sistémicas. Disponible en: http://www.mtas.es/Estadísticas/anuario00/ATE/Index.html

27. Ministerio de Trabajo y Asuntos Sociales .ATE-07. Accidentes en jornadas de trabajo con baja, según gravedad por agente material causante. Disponible en: http://www.mtas.es/estadisticas/ANUARIO2001/HTML/ATE/ate07.html

28. Ministerio de Trabajo y Asuntos Sociales .ATE-07. Accidentes en jornadas de trabajo con baja, según 
gravedad por agente material causante. www.mtas.es/estadisticas/ANUARIO2002/ATE/at e07_1.htm

29. Ministerio de Trabajo y Asuntos Sociales. ATE-38 Enfermedades profesionales según gravedad por tipo de enfermedad. Disponible en: www.mtas.es/estadisticas/ANUARIO2002/ATE/at e38 1.htm

30. Ministerio de Trabajo y Asuntos Sociales. ATE-06. Enfermedades profesionales, según gravedad, por tipo de enfermedad. Disponible en: http:// www.mtas.es/estadisticas/ANUARIO2003/ATE/in dex.htm

31. National Institute for Ocuppational Safety and Health. Nacional occupational Exposure Survey Conducted from 1981 to 1983 . National Institute for Ocuppational Safety and Health; 1989. Disponible en. http://www.cdc.gov/noes/

32. Kauppinen T, Toikkanen J, Pedersen D, Young R, Ahrens W, Boffetta P, Hansen J, Kromhout H, Maqueda Blasco J, Mirabelli D, de la Orden-Rivera V, Pannett B, Plato N, Savela A, Vincent R, Kogevinas M. Occupational exposure to carcinogens in the European Union. Occup Environ Med. 2000;57(1):10-8.

33. Nordic Council of Ministers, Chemical group. Substances in Products in Nordic Countries. CDVersion 2.0 build 1 niv.. Landskronagade 33, 2100 København Ø, Denmark; 2002.

34. OIT. Enciclopedia de la Salud y Seguridad en el Trabajo. Ministerio de Trabajo y Asuntos Sociales de España. Madrid: OIT; 1998; vol IV.p.104.
35. Caisse Regionale d'Assurance Maladie AlsaceMoselle. Système d'évaluation des risques professionnels des produits chimiques dans les entreprises industrielles et artisanales. Strasbourg: Caisse Regionale d'Assurance Maladie Alsace-Moselle; 2001. Disponible en: http://www.cram-alsacemoselle.fr/Prevent/chimie/index.htm

36. International Agency for Research on Cancer. Monographs Programme on the Evaluation of Carcinogenic Risks to Humans. [citado 20 Sept 2003]. Disponible en: http://monographs.iarc.fr/

37. Hunter D, Adams PH. Hunter's Diseases of Occupations. P.A.B. Reffle, P.H. Adams, P.J. Baxter, and W.R. Lee, Editors., 9th Ed. ACGIH; 2000

38. Environmental defense. [citado 20 Sept 2003] Disponible en: http://www.scorecard.org/index.tcl

39. Norma UNE 81900: 1996 EX. Prevención de riesgos laborales. Reglas generales para la implantación de un sistema de gestión de la prevención de riesgos laborales. Madrid: Asociación Española de Normalización y Certificación; 1996.

40. Mancheño C, Izquierdo MA y col. Secretaría de Salud Laboral de la USMR-CC.OO. La evaluación de riesgos laborales en Madrid. Análisis, deficiencias y propuestas. Madrid: Ediciones GPS; 2002.

41. García AM, Gadea R. Estimación de la mortalidad y morbilidad por enfermedades laborales en España. Arch Prev Riesgos Labor 2004; 7 (1):3-8.

42. Morrell S, Kerr Ch, Taylor R, Salkeld G, Corbett S. Best estimate of the magnitude of mortality due to occupational exposure to hazardous substances. Occup Environ Med 1998;55:634-41. 